\title{
UNVERRICHT-LUNDBORG DISEASE
}

The progression of clinical and neurophysiological findings in a Swedish family with 4 siblings with Unverricht-Lundborg disease is reported from the Departments of Pediatrics and Clinical Neurophysiology, University of Gothenburgh, Sweden and Department of Pediatrics, University of Bergen, Norway. Stage 1 was subclinical at the age of 9-11 years with visual blackouts and polyspike EEG activity on photic stimulation. Stage 2 at 12-15 years of age was characterized by increasing segmental, stimulus sensitive myoclonias, occasional nocturnal myoclonic "cascade" seizures, and episodic 4-6 Hz bilateral sharp waves and polyspikes on photic stimulation. Stage 3, a disabling myoclonic stage at the age of 16-18 years showed periodic generalized myoclonias, frequent nocturnal cascade seizures, ataxia, dysarthria, mental deterioration, wheelchair dependency and continuous EEG slow waves with polyspikes and intense myoclonias on photic stimulation. Obstructive apnea was seen at the peak of the cascade seizures, but consciousness was normal and post-ictal drowsiness was absent. 1 sibling died at age 18 with no apparent cause. Other causes of progressive myoclonus epilepsy were excluded. (Kyllerman M et al. Clinical and neurophysiological development of Unverricht-Lundborg disease in four Swedish siblings. Epilepsia Nov/Dec 1991; $\underline{32: 900-909) . ~}$

COMMENT. Unverricht-Lundborg disease is diagnosed by exclusion since no specific biochemical or pathological markers have been detected. This study provides a classification of the stages of the disease together with a description of a typical "cascade" seizure monitored by video electroencephalography.

Another recessively inherited progressive myoclonus epilepsy, Lafora disease, is reported in 4 children from The Department of Neurology, University of Michigan, Ann Arbor, Michigan. Each child presented in adolescence with progressive seizures, myoclonus, dementia and ataxia, and Lafora disease was proven by biopsy. The EEG in 18 studies showed 1) an early stage at age 12 with mild background slowing and trains of $3 \mathrm{~Hz}$ irregular spike wave activity; 2) intermediate stage at age 16 years with faster frequency spike waves up to $12 \mathrm{~Hz}$ and 3 ) late stage 4 years after onset with slow background with overlying beta activity and fast spike waves and polyspikes (Yen $\mathrm{C}$ et al. Longitudinal EEG studies in a kindred with Lafora disease. Epilepsia Nov/Dec 1991; 32:895-899). The EEG in Lafora disease is distinguished by an increased frequency of the spike wave complexes with disease progression. At the onset of seizures the background EEG slowing should suggest a diagnosis other than an idiopathic generalized epilepsy and the progression to prominent fast spike wave complexes appears to be unique. 